\title{
Cluster of invasive Neisseria meningitidis infections on a cruise ship, Italy, October 2012
}

P Stefanelli (paola.stefanelli@iss.it) ${ }^{1}$, C Fazio ${ }^{1,2}$, A Neri, ${ }^{1,2}$, P Isola 3 , S Sani ${ }^{4}$, P Marelli3, C Martinelli3, P Mastrantonio ${ }^{1}$, M G Pompa

1. Department of Infectious, Parasitic and Immune-mediated Diseases, Istituto Superiore di Sanità (ISS), Rome, Italy

2. These authors contributed equally to this work

3. Clinical Pathology Department, Azienda USL 6, Livorno, Italy

4. Infectious Diseases Unit, Azienda USL 6, Livorno, Italy

5. Communicable Disease and International Prophylaxis Unit, Directorate General of Prevention, Ministry of Health, Rome, Italy

Citation style for this article:

Stefanelli P, Fazio C, Neri A, Isola P, Sani S, Marelli P, Martinelli C, Mastrantonio P, Pompa MG. Cluster of invasive Neisseria meningitidis infections on a cruise ship, Italy, October 2012. Euro Surveill. 2012;17(50):pii=20336. Available online: http://www.eurosurveillance.org/ViewArticle.aspx?Articleld=20336

We describe a cluster of four cases of invasive meningococcal disease that occurred on a cruise ship sailing along the Italian coast in October 2012. All four cases were hospitalised with severe illness and one of them died. This report illustrates the importance of rapid implementation of emergency control measures such as administration of prophylaxis to all crew members and passengers to prevent the spread of the disease in such a close environment.

We report a cluster of four cases of meningitis due to infection with serogroup C Neisseria meningitidis ST-11 clonal complex (cc) that occurred on a cruise ship sailing along the Italian coast in October 2012. Meningococcal serogroup C strains, (ST-11 cc) are known to cause invasive disease burden worldwide $[1,2]$. It is also known that these hyperinvasive strains are responsible for a high mortality rate among cases $[3,4]$. These strains caused several outbreaks in France between 2001 and 2003 that led to targeted vaccination campaigns $[5,6]$.

In Italy, serogroup $\mathrm{C}$ is the second most common serogroup ( $16 \%$ of the 118 cases with a known serogroup in 2011 were caused by serogroup ( infection) after the serogroup B ( $64 \%$ of the 118 cases with a known serogroup in 2011 were caused by serogroup B infection) [7]. Meningococci C ST-11 cc have been identified in Italy during the last five years and those characterised as $C: P 1.5-1,10-8: F 3-6: S T-11$, were responsible for both sporadic and outbreak-associated cases [8].

\section{Cluster description}

In early October 2012, four staff members of a cruise ship sailing on the Italian coast were hospitalised on the same day in a local hospital in Tuscany, with clinically suspected meningitis. The four cases were aged between 26 and 47 years and originated from three different countries and three continents. They were all crew members working in the kitchen. One of these four hospitalised patients died; no secondary cases have been reported among other persons present on the ship or among other contacts of the four cases, during the follow-up. Health authorities conducted interviews to determine the travel history of the cases and Naples was established as the last stop before the onset of symptoms. No common source of contamination could be identified during the investigations.

\section{Laboratory investigation}

One day after hospitalisation, the cerebrospinal fluid (CSF) of the four patients was examined by direct microscopy observation at the microbiology laboratory of the Livorno Hospital and the diagnosis of bacterial meningitis was established. Furthermore, on the same day, latex test and culture were performed in the same hospital, whereas serogroup C meningococci were confirmed by rapid molecular test at the Laboratory of the Paediatric Department, Meyer Hospital, University of Florence. The four $N$. meningitidis isolates identified, were sent to the National Reference Laboratory for Invasive Meningococcal Diseases of the Istituto Superiore di Sanità for serogroup confirmation, antimicrobials susceptibility and molecular typing characterisation.

In particular, multilocus sequence typing (MLST) [9] and sequencing of outer membrane proteins PorA and FetA were performed to define the clonal complex and the finetype, respectively; por $B$ gene was also analysed. Variable number tandem repeats (VNTR) typing, using standardised procedures [10], was also carried out to further discriminate among strains. Sequence analysis of penA gene was used to determine the corresponding allele defining isolates with a decreased susceptibility to penicillin [11].

The molecular investigations showed that all strains were C:P1.5-1,10-8:F3-6:ST-11. The discriminatory power of MLST and antigen sequence typing highlighted that the four isolates clearly belonged to ST-11 cc, finetype $\mathrm{P} 1.5-1,10-8: \mathrm{F}_{3}-6$. The porB allele was $2-2$ for all of them. The VNTR analysis (data not shown) confirmed the presence of a unique profile among the four 
strains which was different from all the others found in ST-11 Cc isolates circulating in Italy [12].

The antibiotic susceptibility assay showed a full susceptibility to rifampicin, ciprofloxacin, ceftriaxone and a decreased susceptibility to penicillin and ampicillin. The analysis of penA gene showed the presence of the same allele (248), often associated with a decreased susceptibility to penicillin (0.06<MIC $\leq 1 \mathrm{mg} / \mathrm{L})$ [13].

\section{Control measures}

On the hospital admission day of the four cases described above an alert was launched from the Maritime Port Health Offices of Livorno to the Ministry of Health which ensured rapid communication of this cluster to national and international health authorities for achieving a coordinated response and proper management of this public health event.

On the same day, as an emergency control measure, it was decided to administer chemoprophylaxis with ciprofloxacin to about 2,000 persons present on the ship (international passengers and crew members) before some people left.

In general, the management of meningococcal disease cases focuses on early recognition and antibiotic treatment to reduce case fatality rate and to control the spread of the disease. In the cluster described above, the prompt prophylaxis to all crew members and passengers, as an emergency control measure in such a close environment as a ship, ensured the meningococcal spread control. The rapid strain typing was essential to assess the link among isolates and to highlight the circulation of hyper-invasive ST-11 meningococci.

\section{Acknowledgments}

The authors thank everyone who took part in this investigation: the staff of the Maritime Port Health Offices of Livorno; the staff of the Livorno Hospital; Dr Chiara Azzari, Paediatrician Department, Anna Meyer Hospital, University of Florence, for the rapid identification of serogroup C meningococcal strains on CSFs; Local Health Authorities.

This publication made use of the Neisseria Multi Locus Sequence Typing website [13] developed by Keith Jolley and sited at the University of Oxford (Jolley \& Maiden 2010, BMC Bioinformatics, 11:595). The development of this site has been funded by the Wellcome Trust and European Union.

This work was partially funded by the Ministry of Health-CCM Project fasc.1M12 "Sorveglianza delle Malattie Batteriche Invasive da Neisseria meningitidis, Streptococcus pneumoniae ed Hemophilus influenzae" 2010-2012.
References

1. Watkins ER, Maiden MC. Persistence of Hyperinvasive Meningococcal Strain Types during Global Spread as Recorded in the PubMLST Database. PLoS One. 2012;7(9):e45349.

2. Deghmane AE, Parent du Chatelet, Szatanik M, Hong E, Ruckly C, Giorgini D, et al. Emergence of new virulent Neisseria meningitidis serogroup C Sequence Type 11 isolates in France. Infect Dis. 2010;202(2): 247-50.

3. Zarantonelli ML, Lancellotti M, Deghmane AE, Giorgini $D$, Hong E, Ruckly C, et al. Hyperinvasive genotypes of Neisseria meningitidis in France. Clin Microbiol Infect. 2008;14(5):467-72.

4. Smith I, Caugant DA, Høiby EA, Wentzel-Larsen T, Halstensen A. High case-fatality rates of meningococcal disease in Western Norway caused by serogroup C strains belonging to both sequence type (ST)-32 and ST-11 complexes, 1985-2002. Epidemiol Infect. 2006;134(6):1195-202.

5. Perrocheau A, Taha MK, Lévy-Bruhl D. Epidemiology of invasive meningococcal disease in France in 2003. Euro Surveill. 2005;10(12):pii=587. Available from: http://www. eurosurveillance.org/ViewArticle.aspx?Articleld $=587$

6. Lévy-Bruhl D, Perrocheau A, Mora M, Taha MK, DromellChabrier S, Quatresous I. Vaccination campaign following an increase in incidence of serogroup C meningococcal diseases in the department of Puy-de-Dôme (France). Euro Surveill. 2002;7(5):pii=368. Available from: http://www. eurosurveillance.org/ViewArticle.aspx?Articleld $=368$

7. Istituto Superiore di Sanità (ISS). Dati di sorveglianza delle malattie batteriche invasive aggiornati al 20 agosto 2012. [Surveillance data on invasive bacterial diseases updated 20 August 2012]. Rome: ISS. 25 Sep 2012. Italian. Available from: http://www.simi.iss.it/files/Report_MBI.pdf

8. Fazio C, Neri A, Sofia T, Carannante A, Caporali MG, Salmaso $S$, et al. Characterisation of Neisseria meningitidis C strains causing two clusters in the north of Italy in 2007 and 2008. Euro Surveill. 2009;14(16):pii=19179. Available from: http:// www.eurosurveillance.org/ViewArticle.aspx?Articleld=19179

9. Maiden MC, Bygraves JA, Feil E, Morelli G, Russell JE, Urwin $R$, et al. Multilocus sequence typing: a portable approach to the identification of clones within populations of pathogenic microorganisms. Proc Natl Acad Sci USA. 1998;95(6):3140-5.

10. Yazdankhah SP, Lindstedt BA, Caugant DA. Use of variablenumber tandem repeats to examine genetic diversity of Neisseria meningitidis. J Clin Microbiol. 2005;43(4):1699-705.

11. Taha MK, Vàsquez JA, Hong E, Bennett DE, Bertrand S, Bukovski S, et al. Target gene sequencing to characterize the penicillin G susceptibility of Neisseria meningitidis. Antimicrob Agents Chemother. 2007;51(8):2784-92.

12. Stefanelli P, Fazio C, Sofia T, Neri A, Mastrantonio P. Serogroup $\mathrm{C}$ meningococci in Italy in the era of conjugate menC vaccination. BMC Infect Dis. 2009;9:135.

13. PubMLST [Internet]. Neisseria Sequence Typing Home Page. PubMLST. [Accessed 21 Nov 2012]. Available from: http:// pubmlst.org/neisseria 